\title{
Thermal Effect of Single Loop Shield on High-Voltage Cable Line Capacity
}

\author{
Tkachenko O., Grinchenko V., Dobrodeyev P. \\ State Institution "Institute of Technical Problems of Magnetism \\ of the National Academy of Sciences of Ukraine" \\ Kharkiv, Ukraine
}

\begin{abstract}
The paper deals with a single-loop shield with an asymmetric magnetic coupling used for a magnetic field mitigation of a high-voltage three-phase cable line. The goal is to evaluate a thermal effect of this shield on a cable line capacity. To calculate the flat cable line capacity in the nonshielded case, we use a standard IEC 60287. To achieve the goal we carry out a numerical simulation of the thermal field when the shield is installed. Wherein, we deal with two specific sections. One is a long section with the shield being distant from the cable line. The other is a relatively short section where the shield is located near the power cables. The thermal field is applied for a long section in a two-dimensional formulation, and a three-dimensional formulation is used for the short section. Hence, we have obtained the dependences of the maximum temperature of the power cables on parameters of the shield and its location height above the cable line. The most significant allowable cross-sections of the shield cable and their location height have been determined, when the thermal effect of the shield does not decrease the cable line capacity. These results have ensured the maximum cable line capacity while shielding. The shield temperature is shown to exceed the allowable level in the short section. To reduce it the thermal backfill has been used. We recommend the values of its thermal resistivity to be used for different parameters of the single-loop shield.

Keywords: cable line, capacity, current rating, thermal field, shielding, passive loop.

DOI: https://doi.org/10.52254/1857-0070.2021.1-49.01

UDC: 621.3.017.73:621.315.2.016.2
\end{abstract}

Efectul termic al unui ecran cu o singură buclă asupra capacitate de transmisie a liniei de cablu de înaltă tensiune

Tkachenko A.O., Grinchenko V.S., Dobrodeev P.N.

Instituţia de Stat „Institutul pentru Problemele Tehnice ale Magnetismului al Academiei Naționale de Științe din Ucraina"

Harkov, Ucraina

Rezumat. Lucrarea investighează efectul termic al unui ecran cu un singur circuit cu cuplaj magnetic asimetric, care este utilizat pentru a reduce câmpul magnetic al unei linii de cabluri trifazate de înaltă tensiune. Scopul lucrării este de a evalua impactul unui astfel de ecran asupra randamentului liniei de cablu. În absența unui ecran, debitul este calculat conform IEC 60287. Acest obiectiv este atins prin analiza câmpului termic al unei linii de cablu ecranate prin simulare numerică. În acest caz, sunt luate în considerare două secțiuni caracteristice: o secțiune extinsă, în care ecranul este situat la o anumită distanță de linia de cablu și o secțiune relativ scurtă a ecranului adiacent cablurilor de putere. Se ia în considerare modul de funcționare în regim stabil al liniei de cablu. În consecință, temperaturile cablurilor și ale ecranului nu se schimbă în timp și sunt funcții de coordonate, iar distribuțiile lor respectă ecuația staționară de conducere a căldurii. Distribuția câmpului termic pe o secțiune extinsă este considerată într-o formulare bidimensională și în zona în care ecranul este adiacent cablurilor de alimentare - într-o formulare tridimensională. Ca rezultat, au fost obținute dependențele de temperatura maximă a cablurilor de alimentare de parametrii ecranului și înălțimea locației sale deasupra liniei de cablu. Cele mai semnificative rezultate sunt secțiunile transversale admise ale cablului ecranului și înălțimea locaţiei sale, la care efectul termic al ecranului pe linia cablului nu duce la o scădere a debitului său. Semnificaţia rezultatelor obținute este de a asigura debitul maxim al cablului în timp ce îl protejați.

Cuvinte-cheie: linie de cablu, lăţime de bandă, câmp termic, ecranare, ecran de contur.

\section{Тепловое влияние одноконтурного экрана на пропускную способность высоковольтной кабельной линии \\ Ткаченко А.О., Гринченко В.С., Добродеев П.Н. \\ Государственное учреждение «Институт технических проблем магнетизма Национальной академии наук Украины» \\ Харьков, Украина}

Аннотация. В работе исследовано тепловое влияние одноконтурного экрана с несимметричной магнитной связью, который применяется для уменьшения магнитного поля высоковольтной трехфазной кабельной линии. Целью работы является оценка влияния такого экрана на пропускную способность 
кабельной линии. В случае отсутствия экрана пропускная способность рассчитана согласно стандарту IEC 60287. Поставленная цель достигается за счет анализа теплового поля экранированной кабельной линии путем численного моделирования. При этом рассмотрены два характерных участка: протяженный участок, на котором экран расположен на некотором удалении от кабельной линии, и относительно короткий участок прилегания экрана к силовым кабелям. Рассмотрен установившийся режим работы кабельной линии. Соответственно, температуры кабелей и экрана не меняются с течением времени и являются функциями координат, а их распределения подчиняются стационарному уравнению теплопроводности. Распределение теплового поля на протяженном участке рассмотрено в двумерной постановке, а на участке прилегания экрана к силовым кабелям - в трехмерной постановке. В результате получены зависимости максимальной температуры силовых кабелей от параметров экрана и высоты его расположения над кабельной линией. Наиболее существенными результатами являются найденные допустимые сечения экранного кабеля и высоты его расположения, при которых тепловое влияние экрана на кабельную линию не приводит к снижению ее пропускной способности. Значимость полученных результатов состоит в обеспечении максимальной пропускной способности кабельной линии при ее экранировании. Также показано, что на участке прилегания кабеля экрана к силовым кабелям возможно превышение длительно допустимого значения температуры экрана. В этом случае уменьшение температуры достигается за счет использования засыпки, которая обеспечивает необходимые условия для длительной эксплуатации экрана. Рекомендуемые значения удельного теплового сопротивления засыпки определены при разных параметрах одноконтурного экрана.

Ключевые слова: кабельная линия, пропускная способность, тепловое поле, контурный экран.

\section{ВВЕДЕНИЕ}

$\begin{array}{ccr}\text { Современное } & \text { развитие } & \text { городских } \\ \text { электросетей } & \text { предполагает } & \text { широкое }\end{array}$
использование высоковольтных кабельных линий $35 \div 110$ кВ (КЛ). По сравнению с воздушными линиями электропередачи, КЛ имеют ряд преимуществ. Так, ширина охранной зоны КЛ на порядок меньше ширины охранной зоны воздушной линии. Использование КЛ также позволяет обеспечить внутри домов, расположенных возле трассы линии, выполнение нормативного требования по предельно допустимому уровню магнитного поля (МП). Величины допустимых уровней МП регламентируются национальными нормативами и в разных странах варьируются от десятых долей до десятков микротесла [1]. Например, в Украине для жилых помещений предельно допустимый уровень МП составляет 0,5 мкТл [2]. Для зон жилой застройки нормативные требования традиционно более мягкие (например, 10 мкТл в Украине). В то же время, непосредственно над трассой КЛ норматив может превышаться в несколько раз. Наиболее проблемными с точки зрения уровня создаваемого МП являются зоны муфтовых соединений КЛ, где силовые кабели расположены в одной плоскости и удалены друг от друга на расстояние не менее 0,5 м [2]. МП над этими зонами может в 10 и более раз превышать поле на других участках КЛ. Его снижение до предельно допустимого уровня целесообразно проводить средствами экранирования.
Традиционно для уменьшения МП КЛ используют электромагнитные или магнитные (магнитостатические) экраны [3-7]. Недостатком таких экранов является относительно высокая металлоемкость. Поэтому на данный момент наиболее перспективным методом уменьшения МП КЛ выглядит использование систем контурного экранирования. Простейшим примером является прямоугольный замкнутый электропроводящий контур, эффективность которого экспериментально исследована в [8]. В [9-12] для увеличения эффективности экранирования предлагается использовать систему двух таких контуров. При этом один контур располагается вблизи КЛ, а другой на некоторой высоте между КЛ и уровнем земли. Идея дальнейшего увеличения числа прямоугольных контуров до 5-16 получила развитие в [11-13]. Более технологичным представляется применение дополнительных элементов (конденсаторов либо ферромагнитных сердечников) для увеличения эффективности экранирования. В [9, 10] предложен так называемый «резонансный контурный экран», состоящий из трех параллельно соединенных экранных кабелей с дополнительными конденсаторами в одной или нескольких ветвях. Его недостаток заключается в значительном падении эффективности экранирования при изменении емкости установленных конденсаторов. Наиболее высокими показателями технологичности и эффективности обладают системы типа 
HMCPL [14-16]. В этих системах использованы ферромагнитные сердечники, охватывающие силовые и экранные кабели, для увеличения магнитной связи между КЛ и экранными контурами. В [17] приведен пример практической реализации HMCPL c тремя идентичными сердечниками на действующей КЛ. Недостатком систем такого типа является близкое взаимное расположение экранных и силовых кабелей, что приводит к дополнительному нагреву КЛ и снижению ее пропускной способности.

В [18] для уменьшения МП КЛ предложен новый тип контурного экрана, так называемый одноконтурный экран с ферромагнитными сердечниками и несимметричной магнитной связью, также обеспечивающий сравнительно высокую эффективность экранирования. Особенностью этого экрана, представленного на рис. 1, является удаленность экранного кабеля от КЛ на высоту $H$, равную $0,4 \div 0,6$ расстояния между силовыми кабелями.

Однако недостаточно исследованным остается вопрос теплового воздействия экрана на КЛ, которое может привести к снижению ее пропускной способности. При этом отдельного рассмотрения требует локальный участок прилегания экрана к силовым кабелям. Целью данной работы является оценка влияния одноконтурного экрана с несимметричной магнитной связью на пропускную способность высоковольтной кабельной линии электропередачи.

\section{ПАРАМЕТРЫ КЛ, ГРУНТА И ЭКРАНА}

Предметом исследования настоящей работы является тепловое поле КЛ в зоне расположения одноконтурного экрана с несимметричной магнитной связью.

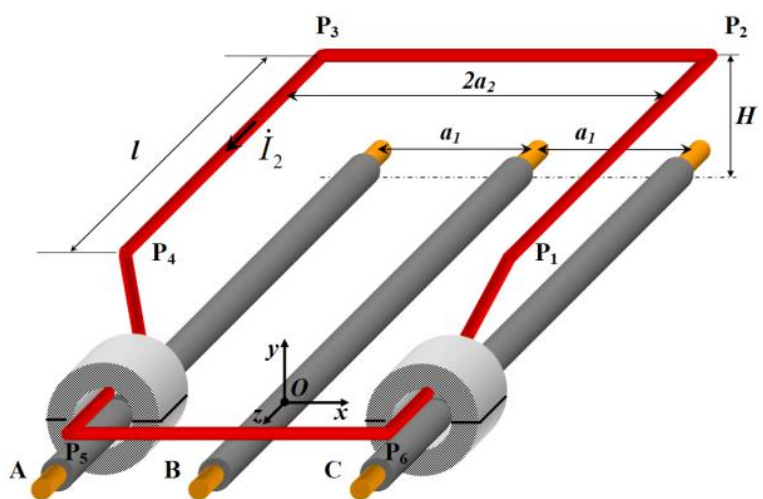

Рис. 1. Конструктивное исполнение одноконтурного экрана с ферромагнитными сердечниками и несимметричной магнитной связью. ${ }^{1}$
Полагается, что КЛ выполнена из типовых силовых кабелей на напряжение 110 кВ АПвЭгаПу-110 [19] с изоляцией из сшитого полиэтилена. В работе рассматриваются кабели с площадью сечения алюминиевой жилы $S_{1}=240$ мм $^{2}$ и $500 \mathrm{mм}^{2}$. Диаметр кабеля с жилой 240 мм $^{2}$ равен 64 мм [19]. Толщина его сшито-полиэтиленовой (СПЭ) изоляции равна $16 \mathrm{мм}$, сечение собственного медного экрана кабеля - 95 мм² $^{2}$ толщина защитного покрытия из поливинилхлорида (ПВХ) равна 6,7 мм. Диаметр кабеля с жилой 500 мм $^{2}$ равен 74 мм [19]. Толщина его изоляции и сечение экрана такие же как у кабеля с жилой 240 мм $^{2}$. Толщина защитного покрытия 7,9 мм.

Длительно допустимая температура жилы кабеля равна $90^{\circ} \mathrm{C}[19]$. При этой температуре удельная проводимость жил полагается равной $2,76 \cdot 10^{7} \mathrm{Cm} / \mathrm{m}[20]$.

При дальнейшем анализе полагается, что силовые кабели состоят из трех частей: жилы, изоляции из СПЭ и защитного покрытия из ПВХ. На основании рекомендаций, изложенных в [21], принято допущение, что собственные экраны кабелей имеют те же тепловые характеристики, что и примыкающие к ним диэлектрические материалы. Соответственно, для собственного экрана кабеля принимается удельное тепловое сопротивление ПВХ 6,0 К·м/Вт. Согласно [21] удельные тепловые сопротивления жилы и СПЭ равны соответственно $4,2 \cdot 10^{-3} \mathrm{~K} \cdot \mathrm{M} / \mathrm{B}$ и и $3,5 \mathrm{~K} \cdot \mathrm{M} / \mathrm{BT}$.

Глубина прокладки кабелей составляет 1,5 м [2]. Грунт полагается однородным и изотропным [22]. Его удельное тепловое сопротивление - 1,2 K·м/Вт [19, 22].

Для одноконтурного экрана с несимметричной магнитной связью используется кабель с алюминиевой жилой и ПВХ изоляцией (также выполняющей роль защитного покрытия) толщиной 3 мм. В большинстве случаев ПВХ изоляция рассчитана на длительно допустимую температуру нагрева $70^{\circ} \mathrm{C}$ [19]. Соответственно, удельная электрическая проводимость жилы экранного кабеля полагается $2,95 \cdot 10^{7} \mathrm{Cm} / \mathrm{m}[20]$.

Как видно из рис. 1 , одноконтурный экран с несимметричной магнитной связью условно можно разделить на две части. Первая часть состоит из параллельных участков $\mathrm{P}_{1} \mathrm{P}_{2}$ и $\mathrm{P}_{3} \mathrm{P}_{4}$, расположенных на высоте $H$ над КЛ, что позволяет минимизировать тепловое 
воздействие тока в экране на КЛ. Длина $l$ этой части экрана на порядок превосходит расстояние $a_{1}$ между силовыми кабелями. Согласно [18] расстояние $2 \cdot a_{2}$ между $\mathrm{P}_{1} \mathrm{P}_{2}$ и $\mathrm{P}_{3} \mathrm{P}_{4}$ принимается равным $3 \cdot a_{1}$ (рис. 1 ). Вторая часть экрана состоит из участков $\mathrm{P}_{4} \mathrm{P}_{5}$ и $\mathrm{P}_{6} \mathrm{P}_{1}$, которые прилегают к силовым кабелям, а также участка $\mathrm{P}_{5} \mathrm{P}_{6}$. Длина этой части в направлении оси $z$ принимается равной 0,5 м, что сопоставимо с ее шириной.

Удельное тепловое сопротивление ферромагнитных сердечников на два порядка меньше чем у грунта. Поэтому они улучшают теплоотвод на участках прилегания экрана к силовым кабелям. Но ввиду незначительности их размера, при дальнейшем анализе, удельное тепловое сопротивление сердечников принимается равным сопротивлению грунта, что соответствует случаю худшего теплового режима КЛ и экрана.

\section{ПРОПУСКНАЯ СПОСОБНОСТЬ КЛ}

В работе рассматривается КЛ с прокладкой кабелей «в плоскости». Промежуток между соседними кабелями на основной трассе КЛ полагается равным диаметру кабеля [2].

Пропускная способность КЛ, при которой температура $\theta_{1}$ жил достигает $90^{\circ} \mathrm{C}$, определяется параметрами силовых кабелей. Согласно стандарту IEC 60287 [20] пропускная способность

$$
I_{1}=\sqrt{\frac{\Delta \theta-W_{d}\left[0,5 T_{1}+m\left(T_{2}+T_{3}+T_{4}\right)\right]}{R T_{1}+m R\left(1+\lambda_{1}\right) T_{2}+m R\left(1+\lambda_{1}+\lambda_{2}\right)\left(T_{3}+T_{4}\right)}},
$$

где $\Delta \theta$ - превышение температуры жил силовых кабелей над температурой окружающей среды, $\mathrm{K} ; R-$ активное сопротивление жилы на единицу длины при $\theta_{1}=90^{\circ} \mathrm{C}$, Ом $/ \mathrm{m} ; W_{d}-$ диэлектрические потери в изоляции жилы на единицу длины, Вт/м; $T_{1}$ - тепловое сопротивление между жилой и собственным экраном кабеля на единицу длины, К·м/Вт; $T_{2}$ - тепловое сопротивление подушки между собственным экраном и броней на единицу длины, К·м/Вт; $T_{3}-$ тепловое сопротивление наружного защитного покрытия кабеля на единицу длины, К·м/Вт; $T_{4}$ - тепловое сопротивление между поверхностью кабеля и окружающей средой, рассчитанное согласно [21], на единицу длины, К·м/Вт; $m=1$ - число жил в кабеле; $\lambda_{1}$ - отношение потерь в собственном экране к общим потерям во всех жилах кабеля; $\lambda_{2}$ - отношение потерь в броне к общим потерям во всех жилах кабеля.

Ток в собственном экране кабеля полагается равным нулю, соответственно, $\lambda_{1}=0$. Поскольку рассматривается небронированный кабель, то $\lambda_{2}=0$ и $T_{2}=0$. Величины диэлектрических потерь и тепловых сопротивлений рассчитываются согласно $[20,21]$. Их значения для кабелей, которые рассматриваются в работе, приведены в табл. 1. Пропускная способность КЛ определяется согласно (1) и при $S_{1}=240 \mathrm{Mм}^{2}$ и $500 \mathrm{Mм}^{2}$ равна $I_{1}=399$ А и $594 \mathrm{~A}$, соответственно.

В зоне муфтовых соединений ток в жилах кабелей равен току на основной трассе КЛ, а расстояние между кабелями увеличивается в несколько раз. Соответственно, температура кабелей в этой зоне, обусловленная тепловым воздействием их токов, уменьшается. В то же время при наличии контурного экрана добавляется тепловое действие его тока на КЛ.

Задача исследования заключается в определении высоты расположения $H$ экранного кабеля и сечения $S_{2}$ его жилы, при которых температура силовых кабелей не превышает $90^{\circ} \mathrm{C}$ на участке расположения экрана.

Мера теплового воздействия экрана на КЛ зависит от действующего значения тока, который в нем протекает. В [18] показано, что максимальная эффективность экранирования МП КЛ достигается при токе в экране

$$
I_{2}=I_{1} \cdot \frac{\sqrt{3}}{2} \cdot \frac{a_{1}}{a_{2}} \cdot \frac{a_{2}^{2}+h_{2}^{2}}{a_{1}^{2}+h_{1}^{2}},
$$

где $h_{2}$ и $h_{1}=h_{2}+H$ - расстояния от плоскости, в которой нормируется МП, до участков $\mathrm{P}_{1} \mathrm{P}_{2}$ и $\mathrm{P}_{3} \mathrm{P}_{4}$ одноконтурного экрана и силовых кабелей, соответственно.

Таблица $1^{2}$

Диэлектрические потери, тепловые сопротивления и пропускная способность ${ }^{3}$

\begin{tabular}{|c|c|c|c|c|c|}
\hline $\begin{array}{c}S_{1}, \\
\mathrm{~mm}^{2}\end{array}$ & $\begin{array}{c}W_{d}, \\
\mathrm{~W} / \mathrm{m}\end{array}$ & $\begin{array}{c}T_{1}, \\
\mathrm{~K} \cdot \mathrm{m} / \mathrm{W}\end{array}$ & $\begin{array}{c}T_{3}, \\
\mathrm{~K} \cdot \mathrm{m} / \mathrm{W}\end{array}$ & $\begin{array}{c}T_{4}, \\
\mathrm{~K} \cdot \mathrm{m} / \mathrm{W}\end{array}$ & $\begin{array}{c}I_{1}, \\
\mathrm{~A}\end{array}$ \\
\hline 240 & 0,172 & 0,580 & 0,223 & 2,07 & 399 \\
\hline 500 & 0,218 & 0,456 & 0,228 & 1,99 & 594 \\
\hline
\end{tabular}


В данной работе рассматривается КЛ в режиме номинальной нагрузки. Поэтому при расчете $I_{2}$ величина $I_{1}$ полагается равной (1). В этом случае тепловыделение в жилах силовых кабелей и экране максимально.

Анализ теплового поля КЛ при использовании одноконтурного экрана с несимметричной магнитной связью выполняется путем численного моделирования. Поскольку часть экрана длиной $l$ является относительно протяженной, анализ теплового поля проводится в двумерной постановке. Длина участка, на котором экран прилегает к КЛ, соизмерима с шириной экрана, a $z$ компонента теплового потока является ненулевой. Поэтому анализ теплового поля проводится в трехмерной постановке.

\section{ДВУМЕРНАЯ ЗАДАЧА}

На участке КЛ, над которым расположена часть экрана длиной $l$, тепловое поле можно считать плоскопараллельным. Поскольку рассматривается работа КЛ в установившемся режиме, то температура $\theta$ не меняется с течением времени и является функцией координат, т.е. $\theta=\theta(x, y)$. Ee распределение подчиняется стационарному уравнению теплопроводности:

$$
\frac{\partial^{2} \theta}{\partial x^{2}}+\frac{\partial^{2} \theta}{\partial y^{2}}=-\rho \cdot q_{\mathrm{V}},
$$

где $\rho$ - удельное тепловое сопротивление подобласти, для которой записывается уравнение, К·м/Вт; $q_{\mathrm{v}}$ - объемная плотность выделяемой тепловой мощности, Вт/м ${ }^{3}$.

В жилах выделяемая тепловая мощность $q_{\mathrm{v}}=(I / S)^{2} / \sigma$, где $I-$ действующее значение тока в жиле силового или экранного кабеля, A; $S$ - сечение жилы, м²; $\sigma$ - электрическая проводимость, См/м.

Уравнение (3) записывается для каждой подобласти расчетной области, показанной на рис. 2: жилы, изоляции и защитного покрытия силовых кабелей, жилы и изоляции экранного кабеля, а также грунта.

На границах подобластей с различными теплофизическими свойствами выполняются условия непрерывности температуры и тепловых потоков:

$$
\theta_{l}=\theta_{r}, \quad \frac{1}{\rho_{l}} \frac{\partial \theta_{l}}{\partial n}=\frac{1}{\rho_{r}} \frac{\partial \theta_{r}}{\partial n},
$$

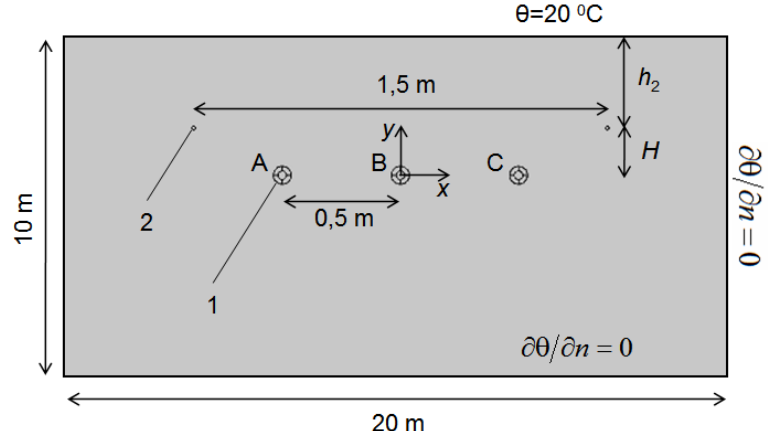

Рис. 2. Расчетная область теплового поля КЛ $\left(1\right.$ - силовой кабель, 2 - экран). ${ }^{4}$

где $\theta_{l}$ и $\theta_{r}-$ соответственно, температура «слева» и «справа» от границы; $\rho_{l}$ и $\rho_{r}-$ удельные тепловые сопротивления соответствующих подобластей; $n$ - вектор нормали.

Анализ литературных источников [23-27] показывает, что для корректного определения теплового поля КЛ целесообразно выбрать прямоугольную расчетную область со сторонами 10 м и 20 м (рис. 2). Верхняя граница расчетной области соответствует уровню поверхности земли. На ней, согласно [25], задается условие первого рода - условие постоянства температуры $\theta=20^{\circ} \mathrm{C}$. На нижней и боковых границах используется условие второго рода - условие теплоизоляции $\partial \theta / \partial n=0$, где $n-$ вектор нормали к границе расчетной области.

Решение поставленной математической задачи выполняется методом конечных элементов в рамках программной среды COMSOL Multiphysics.

При решении в подобластях изоляции и защитного покрытия кабелей наносится сетка типа mapped, в остальных подобластях треугольная. Минимальный размер сетки равен 2,5 мм.

Для верификации результатов моделирования теплового поля были сопоставлены между собой результаты расчетов при двукратном уменьшении размеров сетки и увеличении размеров расчетной области. При этом отличия температур жил силовых кабелей и экрана не превысили $0,5 \%$.

Также было установлено, что результаты моделирования согласуются со стандартом IEC 60287 [20], который позволяет рассчитывать температуру жилы кабеля по действующему значению тока в КЛ. Для этого ток в одноконтурном экране был положен нулевым, а токовая нагрузка КЛ 
варьировалась. Анализ проведен для двух значений удельного теплового сопротивления грунта $(1,0 \mathrm{~K} \cdot \mathrm{M} / \mathrm{BT}$ и $1,2 \mathrm{~K} \cdot \mathrm{M} / \mathrm{BT})$. Во всех случаях отличие температур жил силовых кабелей, найденных путем численного моделирования при различных нагрузках КЛ и нулевом токе в одноконтурном экране, от температур жил, рассчитанных согласно [20] при соответствующих нагрузках, находилось в интервале 1-3\%. Эта погрешность обуславливает отличие пропускной способности $I_{1}=397 \mathrm{~A}, \quad$ найденной путем численного моделирования при $S_{1}=240 \mathrm{Mм}^{2}$, от значения в табл. 1.

Анализ теплового поля КЛ при использовании одноконтурного экрана проведен для ряда значений сечения жилы $S_{2}$ экрана и высоты $H$. Сечение $S_{2}$ лежит в

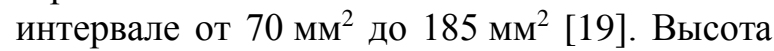
$H$ расположения экрана варьируется от $0,40 \cdot a_{1}$ до $0,60 \cdot a_{1}[18]$.

В табл. 2 и табл. 3 представлены температуры жил центрального силового кабеля $\theta_{1}$ и экрана $\theta_{2}$, полученные в результате численного моделирования. В обеих таблицах выделены допустимые сочетания $S_{2}$ и $H$, при которых $\theta_{1}$ и $\theta_{2}$ не превышают соответствующие допустимые значения.

Результаты, представленные в табл. 2, получены для КЛ, выполненной из кабелей с сечением жил 240 мм² $^{2}$ по которым протекает ток $I_{1}=397 \mathrm{~A}$.

Во всех случаях температура $\theta_{1}$ не превышает допустимые $90^{\circ} \mathrm{C}$. Температура $\theta_{2}$ не превышает допустимые $70^{\circ} \mathrm{C}$, когда сечение экрана $S_{2}$ больше либо равно $95 \mathrm{Mм}^{2}$. Соответственно, экран может устанавливаться на любой высоте от $0,4 \cdot a_{1}$ до $0,6 \cdot a_{1}$. Экран с сечением $S_{2}=70$ мм $^{2}$ может устанавливаться на высоте не менее $H=0,55 \cdot a_{1}$.

Результаты, представленные в табл. 3, получены для КЛ, выполненной из кабелей с сечением жил 500 мм² $^{2}$ по которым протекает ток $I_{1}=594 \mathrm{~A}$.

По сравнению с предыдущим случаем, при одних и тех же $S_{2}$ и $H$, температуры $\theta_{1}$ и $\theta_{2}$ выше. Это обусловлено более высокими значениями токов в силовых кабелях и экране. Как видно из табл. 3 , температуры $\theta_{1}$ и $\theta_{2}$ не превышают соответствующие допустимые значения, если сечение экрана $S_{2}$

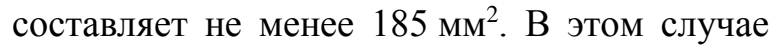
экран может быть установлен на высоте от $0,40 \cdot a_{1}$ до $0,60 \cdot a_{1}$. Экран с сечением

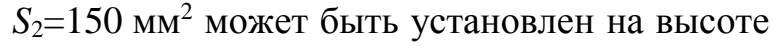
не менее $H=0,55 \cdot a_{1}$.

Полученные результаты показывают, что температура экранного кабеля может превышать допустимые $70^{\circ} \mathrm{C}, \quad$ что накладывает ограничения при выборе его

Температура силового кабеля $\theta_{1}$ и экрана $\theta_{2}$ при $S_{1}=240 \mathrm{Mм}^{2}, I_{1}=397 \mathrm{~A}, a_{1}=0,5$ м 6

\begin{tabular}{|c|c|c|c|c|c|c|}
\hline \multicolumn{2}{|c|}{} & \multicolumn{5}{|c|}{$S_{2}, \mathrm{~mm}^{2}$} \\
\cline { 3 - 7 } \multicolumn{2}{c|}{} & 70 & 95 & 120 & 150 & 185 \\
\hline \multirow{4}{*}{$\Xi$} & $0,40 \cdot a_{1}$ & $87,9 / / 76,0$ & $85,1 / / 65,5$ & $83,5 / / 59,6$ & $82,2 / / 55,3$ & $81,3 / / 52,1$ \\
\cline { 2 - 7 } & $0,45 \cdot a_{1}$ & $87,2 / / 73,5$ & $84,6 / / 63,6$ & $83,0 / / 58,1$ & $81,9 / / 54,0$ & $81,0 / / 51,0$ \\
\cline { 2 - 7 } & $0,50 \cdot a_{1}$ & $86,5 / / 71,0$ & $84,0 / / 61,8$ & $82,6 / / 56,6$ & $81,6 / / 52,7$ & $80,7 / / 49,9$ \\
\cline { 2 - 7 } & $0,55 \cdot a_{1}$ & $85,8 / / 68,7$ & $83,6 / / 60,0$ & $82,2 / / 55,1$ & $81,2 / / 51,5$ & $80,5 / / 48,9$ \\
\cline { 2 - 7 } & $0,60 \cdot a_{1}$ & $85,2 / / 66,4$ & $83,1 / / 58,3$ & $81,9 / / 53,7$ & $81,0 / / 50,3$ & $80,3 / / 47,9$ \\
\hline
\end{tabular}

Таблица $3^{7}$

Температура силового кабеля $\theta_{1}$ и экрана $\theta_{2}$ при $S_{1}=500 \mathrm{mм}^{2}, I_{1}=594 \mathrm{~A}, a_{1}=0,5 \mathrm{M}^{8}$

\begin{tabular}{|c|c|c|c|c|c|c|}
\hline \multicolumn{2}{|c|}{} & \multicolumn{5}{|c|}{$S_{2}, \mathrm{~mm}^{2}$} \\
\cline { 3 - 7 } \multicolumn{2}{c|}{} & 70 & 95 & 120 & 150 & 185 \\
\hline \multirow{4}{*}{$\Xi$} & $0,40 \cdot a_{1}$ & $101,5 / / 121,7$ & $95,2 / / 98,3$ & $91,6 / / 85,1$ & $88,8 / / 75,4$ & $86,7 / / 68,3$ \\
\cline { 2 - 7 } & $0,45 \cdot a_{1}$ & $99,9 / / 116,5$ & $94,0 / / 94,4$ & $90,6 / / 82,0$ & $88,0 / / 72,9$ & $86,1 / / 66,3$ \\
\cline { 2 - 7 } & $0,50 \cdot a_{1}$ & $98,3 / / 111,5$ & $92,9 / / 90,8$ & $89,7 / / 79,1$ & $87,3 / / 70,6$ & $85,5 / / 64,3$ \\
\cline { 2 - 7 } & $0,55 \cdot a_{1}$ & $96,9 / / 106,7$ & $91,8 / / 87,3$ & $88,9 / / 76,3$ & $86,6 / / 68,3$ & $84,9 / / 62,4$ \\
\cline { 2 - 7 } & $0,60 \cdot a_{1}$ & $95,5 / / 102,2$ & $90,8 / / 83,9$ & $88,0 / / 73,7$ & $86,0 / / 66,1$ & $84,4 / / 60,6$ \\
\hline
\end{tabular}


сечения и высоты расположения экрана над КЛ. В то же время, если температура экрана меньше $70^{\circ} \mathrm{C}$, то температура силовых кабелей не превышает допустимого значения $90^{\circ} \mathrm{C}$. Соответственно, пропускная способность КЛ не снижается.

\section{ТРЕХМЕРНАЯ ЗАДАЧА}

Вблизи участков прилегания экрана к КЛ тепловое поле также описывается стационарным уравнением теплопроводности. В отличие от предыдущего случая, уравнение записывается в трехмерной постановке:

$$
\frac{\partial^{2} \theta}{\partial x^{2}}+\frac{\partial^{2} \theta}{\partial y^{2}}+\frac{\partial^{2} \theta}{\partial z^{2}}=-\rho \cdot q_{\mathrm{v}}
$$

Величины удельных тепловых сопротивлений $\rho$ подобластей и объемной плотности $q_{\mathrm{v}}$ выделяемой тепловой мощности даны в предыдущих разделах. Трехмерная система координат показана на рис. 1. Как и при решении двумерной задачи, размер расчетной области по оси $y$ составляет 10 м.

Геометрия одноконтурного экрана и КЛ и создаваемое ими тепловое поле симметричны относительно плоскости $y O z$. Исключение из рассмотрения полупространства $\quad x<0$ позволяет в 2 раза уменьшить размер трехмерной расчетной области. Для этого плоскость $y O z$ выбирается в качестве границы расчетной области, на которой задается условие $\partial \theta / \partial n=0$. При решении двумерной задачи длина расчетной области по оси $x$ составляет 20 м. Соответственно, в данном случае она равна 10 м.

Размер расчетной области по оси $z$ и, соответственно, длина рассматриваемого участка КЛ полагаются равными 10 м. В этом случае на концах расчетной области распределение температуры силовых кабелей по оси z близко к асимптотическому.

В качестве расчетной области выбран куб со стороной 10 м. Точка $O$ начала координат выбрана таким образом, что расчетная область лежит в интервалах $0<x<10$ м, $-8,5 \mathrm{м}<y<1,5 \mathrm{~m},-5 \mathrm{м}<z<5$ м. Участок, на котором экран прилегает к КЛ, лежит в интервале $-0,25 \mathrm{~m}<z<0,25 \mathrm{~m}$, а его центр находится в точке с координатами $x=a_{1}, z=0$.

На верхней границе расчетной области используется условие постоянства температуры $\theta=20^{\circ} \mathrm{C}$. На остальных границах полагается $\partial \theta / \partial n=0$. На границах сред с различными теплофизическими свойствами выполняются условия (4).

На силовые кабели и экран наносится сетка, состоящая из четырехугольных призм, с шагом $0,01 \mathrm{~m}$ по оси $z$ (рис. 3). Минимальный размер элемента сетки равен 1/12 диаметра кабеля. В остальных областях используется сетка, состоящая из тетраэдров.

Для решения трехмерной задачи используется программная среда COMSOL Multiphysics. Верификация корректности расчета выполнена путем сопоставления между собой результатов моделирования теплового поля при двукратном уменьшении размеров сетки.

В случае $S_{1}=240$ мм $^{2}$, как видно из табл. 2 , наибольший нагрев силовых кабелей и экрана наблюдается при $S_{2}=70 \mathrm{Mм}^{2}$ и $H=0,55 \cdot a_{1}$. Рассмотрим нагрев кабелей в области прилегания экрана к КЛ при данных параметрах. На рис. 4 представлены распределения температур силовых кабелей и экрана вдоль оси $z$. Из них следует, что температуры силовых кабелей не превышают допустимого значения.

Также отметим, что в интервале -5 м<z<-4 м температуры силовых кабелей с точностью до $0,5^{\circ} \mathrm{C}$ равны тем, которые были получены путем моделирования теплового поля в рамках двумерной постановки при $a_{1}=0,5$ м в отсутствие одноконтурного экрана. На интервале 2 м <z<5 м температура центрального силового кабеля $\theta_{1}=85,4^{\circ} \mathrm{C}$, что с точностью до $0,5^{\circ} \mathrm{C}$ соответствует значению из табл. 2.

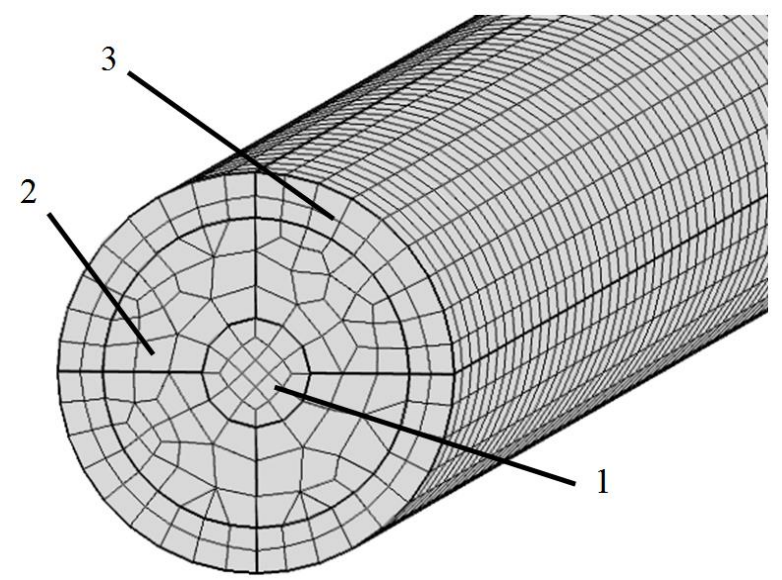

Рис. 3. Расчетная сетка, нанесенная на жилу, изоляцию и защитное покрытие силового кабеля (1 - жила, 2 - изоляция, 3 - защитное покрытие). ${ }^{9}$ 
Также корреспондируются между собой значения температур экрана на кривой 4 (рис. 4) и $\quad$ в табл. 2. Это подтверждает корректность разработанной трехмерной модели теплового поля.

Из рис. 4 следует, что на участках прилегания экрана к КЛ, который находится в интервале $\quad-0,25 \mathrm{~m}<z<0,25 \mathrm{~m}, \quad$ температура $\theta_{2}=84,5^{\circ} \mathrm{C}, \quad$ что превышает длительно допустимую температуру $70^{\circ} \mathrm{C}$.

Аналогичные исследования максимальной температуры в силовых кабелях и экране проведены для всех допустимых сочетаний $S_{2}$ и $H$, которые показаны в табл. 2 и табл. 3. Результаты этих исследований приведены в табл. 4 и табл. 5 , соответственно, для случаев $S_{1}=240 \mathrm{мм}^{2}$ и $500 \mathrm{мм}^{2}$. Из результатов, представленных в табл. 2 - табл. 5, следует, что температура силовых кабелей ниже длительно допустимой $90^{\circ} \mathrm{C}$ на всей длине КЛ.

Таким образом, пропускная способность КЛ при использовании одноконтурного экрана с несимметричной магнитной связью не снижается.

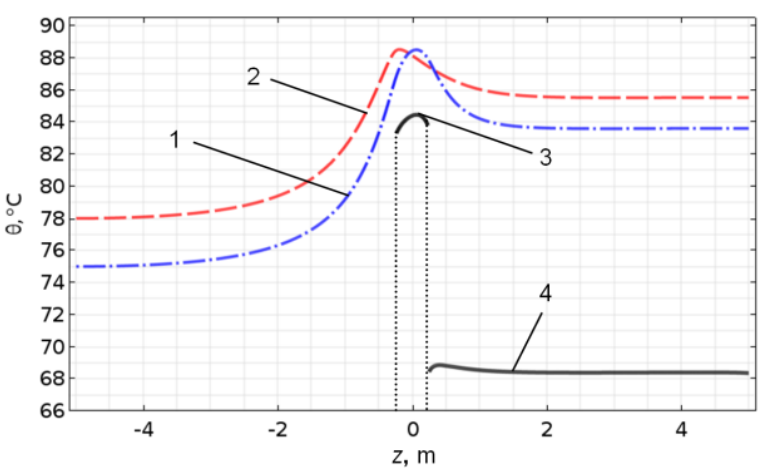

Рис. 4. Распределения температур силовых кабелей и экрана вдоль КЛ при $S_{2}=70$ мм², $H=0,55 \cdot a_{1}$ (1 и 2 - жилы кабелей А и B, 3 - экран прилегает к КЛ, 4 - экран на высоте $H$ над КЛ). ${ }^{10}$

Таблица $4^{11}$

Температура силового кабеля $\theta_{1}$ и экрана $\theta_{2}$ на участках прилегания экрана к КЛ при

\begin{tabular}{|c|c|c|c|c|}
\hline & & \multicolumn{2}{|c|}{$S_{2}, \mathrm{~mm}^{2}$} & \\
\hline & & 70 & 95 & 120 \\
\hline \multirow{5}{*}{$\begin{array}{l}\Xi \\
\Xi\end{array}$} & $0,40 \cdot a_{1}$ & $n / a$ & $87,1 / / 79,3$ & $85,0 / / 73,0$ \\
\hline & $0,45 \cdot a_{1}$ & $n / a$ & $86,6 / / 77,8$ & $84,6 / / 71,9$ \\
\hline & $0,50 \cdot a_{1}$ & $n / a$ & $86,0 / / 76,4$ & $84,1 / / 70,8$ \\
\hline & $0,55 \cdot a_{1}$ & $88,5 / / 84,5$ & $87,4 / / 77,8$ & $83,7 / / 69,7$ \\
\hline & $0,60 \cdot a_{1}$ & $87,9 / / 82,6$ & $85,0 / / 73,8$ & $83,3 / / 68,7$ \\
\hline
\end{tabular}

Таблица $5^{13}$

Температура силового кабеля $\theta_{1}$ и экрана $\theta_{2}$ на участках прилегания экрана к КЛ при

\begin{tabular}{|c|c|c|c|}
\multicolumn{2}{c}{$S_{1}=500 \mathrm{MM}^{2}, I_{1}=594 \mathrm{~A}, a_{1}=0,5 \mathrm{M}^{14}$} \\
\cline { 3 - 4 } \multicolumn{2}{|c|}{} & \multicolumn{2}{c|}{$S_{2}, \mathrm{~mm}^{2}$} \\
\hline \multirow{5}{*}{$\Xi$} & 150 & 185 \\
\hline \multirow{2}{*}{$\approx$} & $0,40 \cdot a_{1}$ & $n / a$ & $88,1 / / 81,6$ \\
\cline { 2 - 4 } & $0,45 \cdot a_{1}$ & $n / a$ & $87,5 / / 80,1$ \\
\cline { 2 - 4 } & $0,50 \cdot a_{1}$ & $n / a$ & $86,9 / / 78,6$ \\
\cline { 2 - 4 } & $0,55 \cdot a_{1}$ & $88,4 / / 83,5$ & $86,3 / / 77,2$ \\
\hline & $0,60 \cdot a_{1}$ & $87,8 / / 81,8$ & $85,8 / / 75,9$ \\
\hline
\end{tabular}

Из табл. 4 и табл. 5 видно, что в некоторых случаях температура экранного кабеля на участках его прилегания к КЛ может превышать допустимые $70^{\circ} \mathrm{C}$. Для ее уменьшения может быть использована засыпка (рис. 5), которая выполняется сыпучим материалом с удельным тепловым сопротивлением $\rho_{b}$, меньшим сопротивления грунта [24-26]. Поскольку уменьшение температуры требуется лишь в ограниченной области, то для засыпки выбран параллелепипед $2 \mathrm{M} \times 1 \mathrm{M} \times 1 \mathrm{~m}$ с центром в точке $O$.

Тепловое поле в области засыпки описывается уравнением (5). Для случая $S_{1}=240 \mathrm{Mм}^{2}, S_{2}=70 \mathrm{Mм}^{2}, H=0,55 \cdot a_{1}$ выполнена серия расчетов при различных значениях $\rho_{b}$. В результате установлено, что удельное тепловое сопротивление засыпки не должно превышать $0,5 \mathrm{~K} \cdot \mathrm{M} /$ Вт. При таком значении $\rho_{b}$, как видно из рис. 6 , максимальная температура экрана не превышает $69^{\circ} \mathrm{C}$. Сравнение распределений, представленных на рис. 4 и рис. 6 , показывает ожидаемое уменьшение температуры силовых кабелей и экрана в области засыпки.

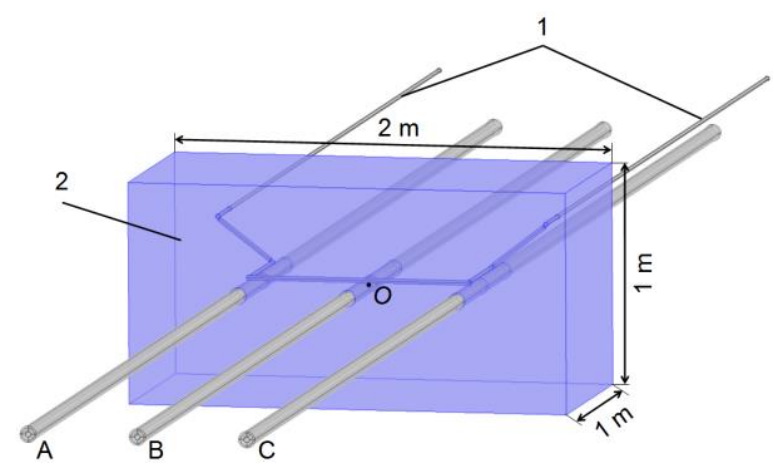

Рис. 5. Область выполнения засыпки для уменьшения температуры экрана на участках его прилегания к КЛ (1 - экран, 2 - засыпка). ${ }^{15}$ 


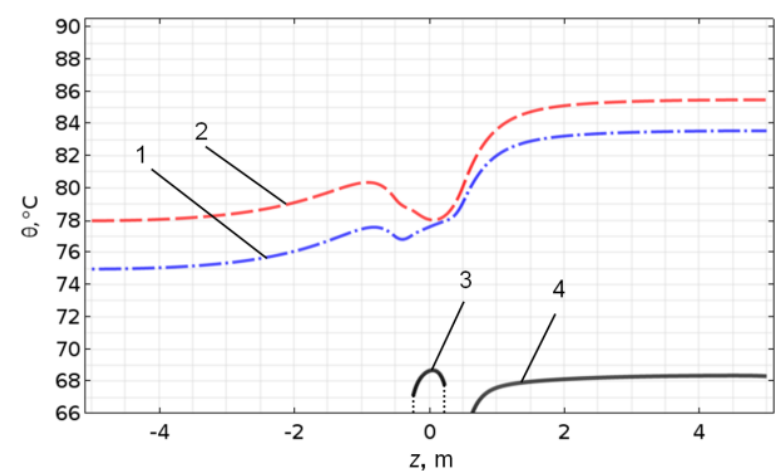

Рис. 6. Распределения температур силовых кабелей и экрана вдоль КЛ при $S_{2}=70$ мм' $H=0,55 \cdot a_{1}$ в случае использования засыпки (1 и 2 - жилы кабелей А и В, 3 - экран прилегает к КЛ, 4 - экран на высоте $Н$ над КЛ). ${ }^{16}$

Вне области засыпки поведение соответствующих температурных распределений на обоих рисунках идентичны. Так, температура протяженной части экрана, расположенной на высоте $H$ над КЛ, практически не меняется при использовании локальной засыпки.

Расчеты, при которых $\rho_{b}$ варьировалось в интервале от $0,5 \mathrm{~K} \cdot \mathrm{m} / \mathrm{BT}$ до $1,2 \mathrm{~K} \cdot \mathrm{m} / \mathrm{BT}$, выполнены при $S_{1}=240 \mathrm{Mм}^{2}$ и $500 \mathrm{мм}^{2}$ для каждой пары значений параметров $S_{2}$ и $H$, рассмотренных в табл. 4 и табл. 5. При этом в каждом случае определена величина $\rho_{b}$, при которой допускается длительная эксплуатация КЛ и экрана. Результаты представлены в табл. 6 и табл. 7.

В табл. 6 для $S_{2}=70 \mathrm{Mм}^{2}$ и в табл. 7 для $S_{2}=150$ мм $^{2}$ не приведены значения $\rho_{b}$ при некоторых значениях $H$. Это обусловлено тем, что применение засыпки в рекомендованной области не позволяет уменьшать температуру протяженного участка экрана, и, соответственно, длительная эксплуатация экрана в этих случаях не допустима.

Также не приведены значения $\rho_{b}$ в табл. 6 для $S_{2}=120 \mathrm{Mм}^{2}$ при $H=0,55 \cdot a_{1}$ и $0,60 \cdot a_{1}$, а также для $S_{2}=150 \mathrm{mм}^{2}$ и $185 \mathrm{mм}^{2}$. В этих случаях засыпка не требуется, поскольку температуры жил силовых кабелей и экрана не превышают допустимые $90^{\circ} \mathrm{C}$ и $70^{\circ} \mathrm{C}$, соответственно.

Из полученных результатов видно, что максимально допустимое удельное тепловое сопротивление засыпки увеличивается при увеличении сечения экрана. Это объясняется снижением тепловой мощности, выделяемой в экране, за счет уменьшения его активного сопротивления.
Таблица $6^{17}$

Максимально допустимое удельное тепловое сопротивление $\rho_{b}(\mathrm{~K} \cdot \mathrm{m} / \mathrm{BT})$ засыпки при

\begin{tabular}{|c|c|c|c|c|}
\multicolumn{2}{c}{$S_{1}=240 \mathrm{MM}^{2}, I_{1}=397 \mathrm{~A}, a_{1}=0,5 \mathrm{M}^{18}$} \\
\hline \multicolumn{2}{|c|}{} & \multicolumn{3}{|c|}{$S_{2}, \mathrm{~mm}^{2}$} \\
\cline { 3 - 5 } \multicolumn{2}{|c|}{} & 70 & 95 & 120 \\
\hline \multirow{4}{*}{$\approx$} & $0,40 \cdot a_{1}$ & $n / a$ & 0,67 & 1,00 \\
\cline { 2 - 5 } & $0,45 \cdot a_{1}$ & $n / a$ & 0,74 & 1,05 \\
\cline { 2 - 5 } & $0,50 \cdot a_{1}$ & $n / a$ & 0,83 & 1,10 \\
\cline { 2 - 5 } & $0,55 \cdot a_{1}$ & 0,50 & 0,87 & $n / a$ \\
\cline { 2 - 5 } & $0,60 \cdot a_{1}$ & 0,57 & 0,90 & $n / a$ \\
\hline
\end{tabular}

Таблица $7^{19}$

Максимально допустимое удельное тепловое сопротивление $\rho_{b}(\mathrm{~K} \cdot \mathrm{m} / \mathrm{BT})$ засыпки при

\begin{tabular}{|c|c|c|c|}
\multicolumn{2}{|c|}{$S_{1}=500 \mathrm{MM}^{2}, I_{1}=594 \mathrm{~A}, a_{1}=0,5 \mathrm{M}^{20}$} \\
\cline { 3 - 4 } \multicolumn{2}{|c|}{} & \multicolumn{2}{c|}{$S_{2}, \mathrm{~mm}^{2}$} \\
\hline \multirow{4}{*}{$\Xi$} & $0,40 \cdot a_{1}$ & $n / a$ & 0,57 \\
\cline { 2 - 4 } & $0,45 \cdot a_{1}$ & $n / a$ & 0,63 \\
\cline { 2 - 4 } & $0,50 \cdot a_{1}$ & $n / a$ & 0,69 \\
\cline { 2 - 4 } & $0,55 \cdot a_{1}$ & 0,50 & 0,75 \\
\cline { 2 - 4 } & $0,60 \cdot a_{1}$ & 0,57 & 0,80 \\
\hline
\end{tabular}

Также допустимое удельное тепловое сопротивление засыпки увеличивается при увеличении высоты установки экрана $H$, что согласно (2) приводит к снижению тока в экране.

Таким образом, применение засыпки объемом $2 \mathrm{M}^{3}$, удельное тепловое сопротивление которой не превышает значения, приведенные в табл. 6 и табл. 7 , обеспечивает условия длительной эксплуатации экрана.

\section{ВЫВОДЫ}

Выполнена оценка теплового влияния одноконтурного экрана с несимметричной магнитной связью на кабельную линию $35 \div 110$ кВ из одножильных кабелей сечением $240 \mathrm{mм}^{2}$ и $500 \mathrm{мм}^{2}$ в зоне муфтовых соединений. Показано, что применение экрана, который выполнен из одножильного низковольтного кабеля допустимых сечений, определенных в работе, и удален от кабельной линии на $0,4 \div 0,6$ расстояния между силовыми кабелями, не приводит к снижению ее пропускной способности.

В области локального перегрева одноконтурного экрана на участках его 
прилегания к силовым кабелям предложено использовать засыпку объемом $2 \mathrm{M}^{3}$ из сыпучих материалов, удельное тепловое сопротивление которых варьируется в диапазоне $0,5 \div 1,1 \mathrm{~K} \cdot \mathrm{M} / \mathrm{BT}$ в зависимости от параметров кабельной линии и экрана.

\section{APPENDIX 1 (ПРИЛОЖЕНИЕ 1)}

${ }^{1}$ Fig. 1. Design of single-loop shield with ferromagnetic cores and asymmetric magnetic coupling.

2,3 Table 1. Dielectric losses, thermal resistances, and current rating of cables.

${ }^{4}$ Fig. 2. Computational domain of cable line thermal field ( 1 - power cable, 2 - shield).

5,6Table 2. Power cable temperature $\theta_{1}$ and shield temperature $\theta_{2}$ when cross-section of cable cores is $S_{1}=240 \mathrm{~mm}^{2}$, rms of cable line current is $I_{1}=397 \mathrm{~A}$, and distance between power cables is $a_{1}=0.5 \mathrm{~m}$.

${ }^{7,8}$ Table 3. Power cable temperature $\theta_{1}$ and shield temperature $\theta_{2}$ when $S_{1}=500 \mathrm{~mm}^{2}, I_{1}=594 \mathrm{~A}$, and $a_{1}=0,5 \mathrm{~m}$.

${ }^{9}$ Fig. 3. Computational mesh applied to core, insulation, and protective coating of power cable (1 - core, 2 - XLPE, 3 - PVC).

${ }^{10}$ Fig. 4. Temperature distributions in power cables and shield along cable line when cross-section of shield core is $S_{2}=70 \mathrm{~mm}^{2}$, height of shield installation is $H=0,55 \cdot a_{1}$ ( 1 and 2 - cores of cables $\mathrm{A}$ and $\mathrm{B}$, 3 - shield section near cable line, 4 - shield above cable line at height $H$ ).

11,12 Table 4. Power cable temperature $\theta_{1}$ and shield temperature $\theta_{2}$ in convergence section with $S_{1}=240 \mathrm{~mm}^{2}, I_{1}=397 \mathrm{~A}$, and $a_{1}=0,5 \mathrm{~m}$.

${ }^{13,14}$ Table 5. Temperature of power cable $\theta_{1}$ and shield $\theta_{2}$ in convergence section when $S_{1}=500 \mathrm{~mm}^{2}$, $I_{1}=594 \mathrm{~A}$, and $a_{1}=0,5 \mathrm{~m}$.

${ }^{15}$ Fig. 5. Backfill used to reduce shield temperature in convergence section ( 1 - shield, 2 - backfill).

${ }^{16}$ Fig. 6. Temperature distributions in power cables and shield along cable line in backfilling case when $S_{2}=70 \mathrm{~mm}^{2}, H=0,55 \cdot a_{1}$ ( 1 and 2 - cores of cables A and $\mathrm{B}, 3$ - shield section near cable line, 4 - shield above cable line at height $H$ ).

${ }^{17,18}$ Table 6. Maximum allowable thermal resistivity $\rho_{b}(\mathrm{~K} \cdot \mathrm{m} / \mathrm{W})$ of backfill when $S_{1}=240 \mathrm{~mm}^{2}, I_{1}=397 \mathrm{~A}$, and $a_{1}=0,5 \mathrm{~m}$.

${ }^{19,20}$ Table 7. Maximum allowable thermal resistivity $\rho_{b}(\mathrm{~K} \cdot \mathrm{m} / \mathrm{W})$ of backfill when $S_{1}=500 \mathrm{~mm}^{2}, I_{1}=594 \mathrm{~A}$, and $a_{1}=0,5 \mathrm{~m}$.

\section{Литература (References)}

[1] World Health Organization. Global Health Observatory data repository. Exposure limits for low-frequency fields (public). Available at: https://apps.who.int/gho/data/node.main.EMFLI MITSPUBLICLOW?lang=en

(accessed 21.01.2021)
[2] Electrical installation regulations. Kharkiv, Fort Publ., 2017, 760 p. (In Ukrainian).

[3] del-Pino-López J.C., Cruz-Romero P., SerranoIribarnegaray L., Martínez-Román J. Magnetic field shielding optimization in underground power cable duct banks. Electric power systems research, 2014, vol. 114, pp. 21-27. doi: 10.1016/j.epsr.2014.04.001

[4] Sergeant P., Koroglu S. Electromagnetic losses in magnetic shields for buried high voltage cables. Progress in electromagnetics research, 2011, vol. 115, pp. 441-460. doi: 10.2528/PIER11022206

[5] Cardelli E., Faba A., Pirani A. Nonferromagnetic open shields at industrial frequency rate. IEEE transactions on magnetics, 2010, vol. 46, no. 3 , pp. 889-898. doi: 10.1109/TMAG.2009.2031110

[6] Zucca M., Lorusso G., Fiorillo F., Roccato P.E., Annibale M. Highly efficient shielding of highvoltage underground power lines by pure iron screens. Journal of magnetism and magnetic materials, 2008, vol. 320, no. 20, pp. 1065-1069. doi: 10.1016/j.jmmm.2008.04.096

[7] De Wulf M., Wouters P., Sergeant P., Dupre' L., Hoferlin E., Jacobs S., Harlet P. Electromagnetic shielding of high-voltage cables. Journal of magnetism and magnetic materials, 2007, no. 316, pp. 908-911. doi: 10.1016/j.jmmm.2007.03.137

[8] Wall C.A., Arnera P.L., Barbieri M.B. Implementation of a tool for magnetic field mitigation using passive loops. 2012 Sixth IEEE/PES Transmission and Distribution: Latin America Conference and Exposition (T\&D-LA), 2012, pp. 1-5. doi: $10.1109 /$ TDCLA.2012.6319095

[9] del-Pino-López J.C., Cruz-Romero P. The effectiveness of compensated passive loops for mitigating underground power cable magnetic fields. IEEE Transactions on Power Delivery, 2011, vol. 26, no. 2, pp. 674-683. doi: 10.1109/TPWRD.2009.2039150

[10] Cruz P., Hoeffelman J., del Pino J.C. Mitigación de campos magnéticos en líneas subterráneas de potencia mediante el empleo de lazos pasivos. IEEE Latin America Transactions, 2008, vol. 6, no. 2, pp. 59-65.

[11] Maioli P., Zaccone E. Thermal design of HV electric systems with EMF mitigation devices. Conference Conseil International des Grands Réseaux Électriques, 2011, pp. 1-10.

[12] Maioli P., Zaccone E. Passive loops technique for electromagnetic fields mitigation: applications and theoretical considerations. Jicable International Conference on Insulated Power Cables, 2007, pp. 231-236.

[13] Maioli P. Shielding of high voltage cables: Patent No.: US 8,895,851 B2, 2014.

[14] Canova A., Bavastro D., Freschi F., Giaccone L., Repetto M. Magnetic shielding solutions for the 
junction zone of high voltage underground power lines. Electric Power Systems Research, 2012, vol. 89, pp. 109-115. doi: 10.1016/j.epsr.2012.03.003

[15] Canova A., Freschi F., Giaccone L., Guerrisi A., Repetto M. Magnetic field mitigation by means of passive loop: technical optimization. COMPEL: The International Journal for Computation and Mathematics in Electrical and Electronic Engineering, 2012, vol. 31, no. 3, pp. 870-880. doi: 10.1108/03321641211209762

[16] Canova A., Giaccone L. A novel technology for magnetic-field mitigation: high magnetic coupling passive loop. IEEE Transactions on Power Delivery, 2011, vol. 26, no. 3, pp. 16251633. doi: 10.1109/TPWRD.2010.2099671

[17] Canova A., Giaccone L. Magnetic mitigation of HV cable junction zone. 8th International Conference on Insulated Power Cables, 2011, Paper B.1.6.

[18] Rozov V.Yu., Grinchenko V.S., Yerisov A.V., Dobrodeyev P.N. Efficient shielding of threephase cable line magnetic field by passive loop under limited thermal effect on power cables. Electrical engineering \& electromechanics, 2019, no. 6, pp. 50-54. doi: 10.20998/2074272X.2019.6.07

[19] Medium and high voltage XLPE-insulated power cables. PJSC YUZHCABLE WORKS, 56 p.

[20] IEC 60287 "Electric cables - Calculation of the current rating", Part 1-1 "Current rating equations (100\% load factor) and calculation of losses."

[21] IEC 60287 "Electric cables - Calculation of the current rating", Part 2-1 "Thermal resistance Calculation of thermal resistance."

\section{Сведения об авторах.}

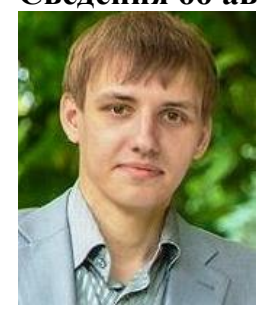

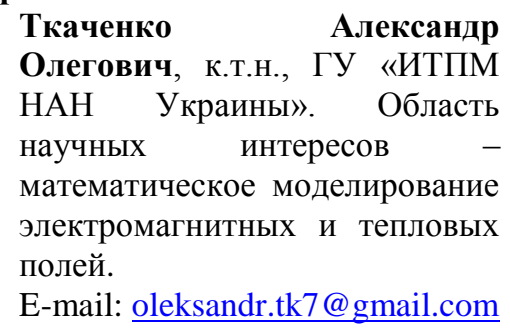

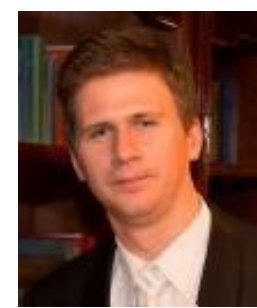

\footnotetext{
Гринченко

Владимир Сергеевич, к.т.н., ГУ «ИТПМ НАН Украины». Область научных интересов - системы экранирования магнитного поля, теоретическая электротехника. E-mail: vsgrinchenko@gmail.com
}

[22] Tykocki, J., Yue, Y., \& Jordan, A. Analysis of thermal field in $110 \mathrm{kV}$ cable systems. Przeglad Elektrotechniczny, 2012, pp. 142-145.

[23] Aras F., Oysu C., Yilmaz G. An assessment of the methods for calculating ampacity of underground power cables. Electric Power Components and Systems, 2005, vol. 33, no. 12, pp. 1385-1402. doi: 10.1080/15325000590964425

[24] Ocłoń P., Cisek P., Pilarczyk M. and Taler D. Numerical simulation of heat dissipation processes in underground power cable system situated in thermal backfill and buried in a multilayered soil. Energy Conversion and Management, 2015, vol. 95, pp. 352-370. doi: 10.1016/j.enconman.2015.01.092

[25] Ocłoń P., Cisek P., Rerak M., Taler D., Rao R.V., Vallati A. and Pilarczyk M. Thermal performance optimization of the underground power cable system by using a modified Jaya algorithm. International Journal of Thermal Sciences, 2018, vol. 123, pp. 162-180. doi: 10.1016/j.ijthermalsci.2017.09.015

[26] Rerak M., Ocłoń P. Thermal analysis of underground power cable system. Journal of Thermal Science, 2017, vol. 26, no. 5, pp. 465471. doi: 10.1007/s11630-017-0963-2

[27] Battistelli L., Geri A., Veca G.M. Magnetic field shielding of underground power cables in urban areas: a real-life example. WIT transactions on modelling and simulation, 2005, vol. 39, pp. 527540.

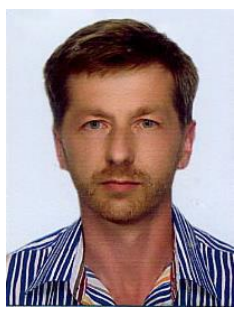

Добродеев

Павел

Николаевич, к.т.н., ученый секретарь ГУ «ИТПМ НАН Украины». Область научных интересов - магнетизм технических объектов.

E-mail: dobrodeyev@nas.gov.ua 